\title{
ON ENDOMORPHISMS OF A SOLENOID
}

\author{
A. M. WILSON
}

\begin{abstract}
Geometrically simple Bernoulli generators are constructed for certain ergodic endomorphisms of solenoids. An arbitrary ergodic solenoidal group automorphism is obtained as the limit of a sequence of such Bernoulli factors and hence, by a theorem of D. S. Ornstein, must be measuretheoretically isomorphic to a Bernoulli shift.
\end{abstract}

In his survey paper [6], B. Weiss stated that, using Y. Katznelson's methods, he can prove that every ergodic automorphism of a solenoid is isomorphic to a Bernoulli shift. The aim of this note is to give an alternative proof of this result, with a partial result in the endomorphism case.

The methods used are similar to those of L. M. Abramov [1], who used geometrically simple generating partitions in order to compute the entropy of certain solenoidal automorphisms. A comparison will show that Abramov's generators are refinements of the Bernoulli generators exhibited in $\$ 2$ below.

The author would like to acknowledge S. M. Rudolfer, who supervised this work, and R. K. Thomas who suggested the problem to us.

For brevity, a working knowledge of measure theory, ergodic theory and topological groups is assumed in what follows, apart from the following fundamental definition of a Bernoulli shift.

A measure preserving map $\phi$ from a separable measure space $(X, \mu)$ to itself will be called a (one-sided) Bernoulli shift if there is a measurable partition $P$ of $X$ (called a Bernoulli generator for $\phi$ ) such that

(i) $\left\{\phi^{-i} P\right\}, i \geqq 0$, is an independent family of partitions, and

(ii) $\vee_{i=0}^{\infty} \phi^{-i} P$ is the point partition of $X$.

If $\phi$ is invertible, (ii) becomes (ii) $\vee_{-\infty}^{\infty} \phi^{i} P$ is the point partition of $X$.

1. Details of solenoids are well documented (see e.g. [1], [3] and [2, Chapter VIII). The following brief characterisation will be subsequently useful.

DEFINITION 1.1. Let $G$ be a noncyclic subgroup of the discrete additive group $Q$ of rational numbers. The character group $\Sigma$ of $G$, called a (onedimensional) solenoid, is a compact, separable, commutative topological group.

Proposition 1.2. Let $\mathbf{a}=\left(a_{1}, a_{2}, \ldots\right)$ be a sequence of integers $a_{i} \geqq 2$. Let $G_{\mathbf{a}}$ be the subgroup of $Q$ generated by the elements $\prod_{i=1}^{n} 1 / a_{i}$, for $n \geqq 1$. Up to isomorphism, every additive subgroup $G$ of $Q$, as in 1.1 , can be represented as a $G_{\mathbf{a}}$ for some such $\mathbf{a}$. If $\mathbf{a}$ is the constant sequence on some integer $a$, then $G_{\mathbf{a}}$ is the group of a-ary rationals, denoted $G_{a}$.

Received by the editors December 4, 1974.

AMS (MOS) subject classifications (1970). Primary 28A65; Secondary 22D40, $22 \mathrm{D} 45$.

Key words and phrases. Solenoid, topological group endomorphism, Bernoulli shift, natural extension, generalised Bernoulli shift. 
The character group of $G_{\mathbf{a}}$ will be denoted $\Sigma_{\mathbf{a}}$. The character group of $G_{a}$ will be denoted $\Sigma_{a}$, called the $a$-adic solenoid. The solenoid $\Sigma_{\mathbf{a}}$ can be viewed as a subgroup of the countable product of circle groups $S$, where $\mathbf{x}=\left(x_{0}, x_{1}, \ldots\right)$ $\in \Sigma_{\mathbf{a}}$ if and only if $\forall i \geqq 0, x_{i} \in S$ and $x_{i+1}^{a_{i+1}}=x_{i}$.

Proposition 1.3. Each endomorphism of $G_{\mathbf{a}}$ is of the form $\psi_{m / n}$, acting as multiplication by $m / n$. By convention, $n>0$. If $m / n \neq 0$, then $m$ and $n$ are coprime (by convention), and $G_{n}$ is a subgroup of $G_{\mathbf{a}}$. If $\psi_{m / n}$ is invertible, its inverse is $\psi_{n / m}$, and $G_{m n}$ is a subgroup of $G_{\mathbf{a}}$.

The endomorphisms of $\Sigma_{\mathbf{a}}$ are in 1-1 correspondence with those of $G_{\mathbf{a}}$, so to each endomorphism $\psi_{m / n}$ the dual endomorphism $\phi_{m / n}$ of $\Sigma_{\mathbf{a}}$ is associated. The nontrivial endomorphisms $\psi_{m / n}$ (i.e. $m / n \neq 0$ ) of $G_{\mathrm{a}}$ are injective,so their duals acting on $\Sigma_{\mathbf{a}}$ are all surjective, Haar measure-preserving endomorphisms.

Proposition 1.4. The endomorphism $\phi_{m / n}$ of $\Sigma_{\mathbf{a}}$ is ergodic if and only if $m / n \neq 0, \pm 1$.

2. Let $\Sigma_{\mathrm{a}}$ be a fixed solenoid, and $\phi_{m / n}$ an ergodic endomorphism of it. Up to isomorphism, $\Sigma_{\mathbf{a}}$ may be represented by $\mathbf{a}=\left(n b_{1}, n b_{2}, \ldots\right)$ where each $b_{i}$ is a positive integer coprime to $n$. Then

$$
\begin{aligned}
\phi_{m / n}\left(x_{0}, x_{1}, \ldots\right) & =\phi_{m / n}\left(x_{1}^{n b_{1}}, x_{2}^{n b_{2}}, \ldots\right) \\
& =\left(x_{1}^{(m / n) \cdot n b_{1}}, x_{2}^{(m / n) \cdot n b_{2}}, \ldots\right)=\left(x_{1}^{m b_{1}}, x_{2}^{m b_{2}}, \ldots\right) .
\end{aligned}
$$

Before exhibiting Bernoulli partitions for such endomorphisms, some notation and a general lemma must be introduced.

Notation. (i) Let $(S, \nu),\left(\Sigma_{\mathrm{a}}, \mu\right)$ denote the circle and solenoidal groups, respectively, with normalised Haar measures.

(ii) For each nonzero integer $N$, let $S(N)$ be the partition of $S$ into $|N|$ arcs $\left\{S_{1}(N), \ldots, S_{|N|}(N)\right\}$ where, for $1 \leqq j \leqq|N|, S_{j}(N)=\{x=\exp 2 \pi i \theta:-j$ $-1 /|N| \leqq \theta<j /|N|\}$.

Observe that translation by any $N$ th root of unity permutes the elements of $S(N)$.

(iii) For $i \geqq 0$, let the $i$ th coordinate projection $\pi_{i}: \Sigma_{\mathbf{a}} \rightarrow S$ be the measurepreserving map given by $\pi_{i}\left(x_{0}, x_{1}, \ldots\right)=x_{i}$.

(iv) For nonzero integers $h$, define $\omega_{h}: S \rightarrow S$ to be the measure preserving endomorphism given by $\omega_{h}(x)=x^{h}$.

(v) We shall say that a partition $P$ of a group $X$ is regular with respect to a subgroup $K$ of $X$ if for each atom $P_{i} \in P$, the sets $\left\{x P_{i}\right\}, x \in K$, are disjoint and form the partition $P$ of $X$.

(vi) For each $r \geqq 1$, the product $\prod_{j=1}^{r} b_{j}$ will be abbreviated to $B_{r}$, and by convention $B_{0}=1$.

LEMMA 2.1. Let $f, g$ be two surjective endomorphisms of a compact separable topological group $X$, with normalised left-invariant Haar measure $\mu$, and let $P$ be a measurable partition of $X$ such that

(i) the elements of $\operatorname{Ker} f$ and $\operatorname{Ker} g$ commute,

(ii) $g(\operatorname{Ker} f)=\operatorname{Ker} f$,

(iii) $P$ is regular with respect to $\operatorname{Ker} f$. 
Then for any measurable partition $Q$ of $X$, the partitions $f^{-1} Q$ and $g^{-1} P$ are independent.

Proof. Since $X$ is compact, $\operatorname{Ker} f$ is finite, say of order $p$. The maps $f$ and $g$ must preserve $\mu$. By (iii), $\mu P_{i}=1 / p$ for $1 \leqq i \leqq p$.

It follows from (iii) that left translation by any element of $\operatorname{Ker} f$ permutes the elements of $P$, and that the restriction of $f$ to each $P_{i}$ is a bijection onto $X$.

Let $P_{i}, Q_{j}$ be elements of $P, Q$, respectively. It must be shown that

$$
\mu\left(g^{-1} P_{i} \cap f^{-1} Q_{j}\right)=\mu\left(g^{-1} P_{i}\right) \cdot \mu\left(f^{-1} Q_{j}\right)=(1 / p) \mu Q_{j} .
$$

Let $\tilde{P}, \tilde{Q}$ be regular partitions of $g^{-1} P_{i}, f^{-1} Q_{j}$ with respect to $\operatorname{Ker} f, \operatorname{Ker} g$, respectively. (The existence and measurability of such partitions is a simple consequence of Zorn's lemma.)

Then for each atom $\tilde{Q}_{j}$ of $\tilde{Q}$,

$$
f^{-1} Q_{j}=\bigcup_{x \in \operatorname{Ker} f} x \tilde{Q}_{j} \text { and } \mu \tilde{Q}_{j}=(1 / p) \mu Q_{j}
$$

Thus

$$
\begin{aligned}
\mu\left(g^{-1} P_{i} \cap f^{-1} Q_{j}\right) & =\sum_{x \in \operatorname{Ker} f} \mu\left(g^{-1} P_{i} \cap x \tilde{Q}_{j}\right) \\
& =\sum_{x \in \operatorname{Ker} f} \mu\left(x^{-1} \cdot g^{-1} P_{i} \cap \tilde{Q}_{j}\right) .
\end{aligned}
$$

Now

$$
\begin{aligned}
\bigcup_{x \in \operatorname{Ker} f} x^{-1} \cdot g^{-1} P_{i} & =\bigcup_{x \in \operatorname{Ker} f} x\left(\bigcup_{y \in \operatorname{Ker} g} y \cdot \tilde{P}_{i}\right) \\
& =\bigcup_{y \in \operatorname{Ker} g} y\left(\bigcup_{x \in \operatorname{Ker} f} x \tilde{P}_{i}\right) \text { by (i) }
\end{aligned}
$$

where $\tilde{P}_{i}$ is any atom of $\tilde{P}$. But

$$
\begin{aligned}
g\left(\bigcup_{x \in \operatorname{Ker} f} x \tilde{P}_{i}\right) & =\bigcup_{x \in \operatorname{Ker} f} g(x) P_{i} \text { since } g\left(\tilde{P}_{i}\right)=P_{i} \\
& =\bigcup_{x \in \operatorname{Ker} f} x P_{i} \text { by (ii) } \\
& =X .
\end{aligned}
$$

Hence $\cup_{x \in \operatorname{Ker} f} x \tilde{P}_{i}$ contains an atom of a regular partition of $X$ with respect to $\operatorname{Ker} g$, therefore $\cup_{x \in \operatorname{Ker} f} x^{-1} \cdot g^{-1} P_{i}=X$.

But $\mu\left(g^{-1} P_{i}\right)=\mu P_{i}=1 / p$, so the sets $\left\{x^{-1} \cdot g^{-1} P_{i}\right\}$ are disjoint as $x$ varies over Ker $f$. Therefore

$$
\mu\left(g^{-1} P_{i} \cap f^{-1} Q_{j}\right)=\mu \tilde{Q}_{j}=(1 / p) \mu Q_{j} \text {. Q.E.D. }
$$

Proposition 2.2. Let $\phi_{m / n}$ be an ergodic endomorphism of $\Sigma_{\mathbf{a}}$, as above. Then for both $N=m$ and $N=n$, the partition $P=\pi_{0}^{-1} S(N)$ is a Bernoulli partition for $\phi_{m / n}$. 
Proof. It will be sufficient to show that for each $r \geqq 0,\left\{\phi_{m / n}^{-i} P\right\}, 0 \leqq i$ $\leqq r$, is an independent family of partitions.

Observe

$$
\begin{aligned}
\bigvee_{i=0}^{r} \phi_{m / n}^{-i} P & =\bigvee_{i=0}^{r} \pi_{i}^{-1} \omega_{B_{i}}^{-1} \omega_{m}^{-i} S(N) \\
& =\pi_{r}^{-1} \omega_{B_{r}}^{-1}\left(\bigvee_{i=0}^{r} \omega_{m}^{-i} \omega_{n}^{-r+i} S(N)\right)
\end{aligned}
$$

By the symmetry of (1), there is no loss of generality in assuming $N=m$. Then

$$
\bigvee_{i=0}^{r} \omega_{m}^{-i} \omega_{n}^{-r+i} S(N)=\omega_{n}^{-r} S(m) \vee \omega_{m}^{-1} \bigvee_{i=1}^{r} \omega_{m}^{-i+1} \omega_{n}^{-r+i} S(m)
$$

In Lemma 2.1 above, set $f=\omega_{m}, g=\omega_{n}^{r}, P=S(m)$ and

$$
Q=\bigvee_{i=1}^{r} \omega_{m}^{-i+1} \omega_{n}^{-r+i} S(m)
$$

Since $S$ is commutative, (i) is satisfied. Condition (ii) follows from the coprimeness of $m$ and $n^{r}$, and (iii) holds by definition of $S(m)$. Hence $\omega_{n}^{-r} S(m)$ and $\omega_{m}^{-1}\left(\bigvee_{i=1}^{r} \omega_{m}^{-i+1} \omega_{n}^{-r+i} S(m)\right)$ are independent partitions of $S$. But $\pi_{r}$ and $\omega_{B r}$ preserve measure, so $P$ and $\vee_{i=1}^{r} \phi_{m / n}^{-i} P$ are independent. The proof is completed by induction.

The rest of this section concerns the cases in which the Bernoulli partitions obtained above are generators.

Proposition 2.3. Let $\phi_{m / n}$ be an ergodic endomorphism of $\Sigma_{\mathbf{a}}$, with $|m|>n$ $>0$. Then $P=\pi_{0}^{-1} S(m)$ is a generator for $\phi_{m / n}$ if either (i) $\phi_{m / n}$ is not invertible and $\Sigma_{\mathrm{a}}=\Sigma_{n}$, or (ii) $\phi_{m / n}$ is invertible and $\Sigma_{\mathrm{a}}=\Sigma_{m n}$.

Proof. (i) Suppose $\phi_{m / n}$ is not invertible. Let $\mathbf{a}=\left(n b_{1}, n b_{2}, \ldots\right)$ where each $b_{i}$ is coprime to $n$. Set $M=|m|$.

(1) Let $\mathbf{x}$ and $\mathbf{y}$ lie in the same atom of $\bigvee_{i=0}^{\infty} \phi_{m / n}^{-i} P$. In particular, for each $r \geqq 0, x_{r}$ and $y_{r}$ lie in the same atom of $\omega_{B_{r}}^{-1} \bigvee_{i=0}^{r} \omega_{m}^{-i} \omega_{n}^{-r+i} S(m)($ as in 2.2(1)).

(2) It can be shown by induction that each atom of $\bigvee_{i=0}^{r} \omega_{m}^{-i} \omega_{n}^{-r+i} S(m)$ $\vee S\left(n^{r}\right)$ is contained in a single arc of $\nu$-measure at most $1 / M^{r+1}$.

For $u, v \in S$, let $\|u-v\|$ be the $\nu$-measure of the shorter arc joining $u$ and $v$.

Then by (1) and (2) above, for each integer $i$ with $0 \leqq i \leqq r$, there is an integer $s(r, i)$ with $0 \leqq s(r, i)<B_{r} M^{i} n^{r-i}$ such that

$$
0 \leqq\left\|x_{r}-y_{r}\right\|-s(r, i) / B_{r} M^{i} n^{r-i} \leqq 1 / B_{r} M^{r+1} .
$$

Setting $i=0$ in (3) gives $0 \leqq\left\|x_{r}-y_{r}\right\|-s(r, 0) / B_{r} n^{r} \leqq 1 / B_{r} M^{r+1}$.

But $x_{r}^{B_{r} n^{r}}=x_{0}, y_{r}^{B_{r} n^{r}}=y_{0}$, so $0 \leqq\left\|x_{0}-y_{0}\right\| \leqq n^{r} / M^{r+1}$. This holds for all $r \geqq 0$, and $M>n$, hence $x_{0}=y_{0}$.

Suppose $x_{i}=y_{i}$ for $0 \leqq i \leqq r-1$. Then $\left\|x_{r}-y_{r}\right\|=\alpha / b_{r} n$ for some integer $\alpha$ with $0 \leqq \alpha<b_{r} n$. Setting $i=r$ in (3) gives

$$
0 \leqq \alpha / n b_{r}-s(r, r) / B_{r} M^{r} \leqq 1 / B_{r} M^{r+1} \text {. }
$$


Hence $0 \leqq B_{r-1} M^{r} \alpha-n s(r, r) \leqq n / M<1$, which implies $B_{r-1} M^{r} \alpha=$ $n \cdot s(r, r)$.

But since $B_{r-1}$ and $m$ are coprime to $n$, it follows that $n$ divides $\alpha$. Moreover, if $b_{r}=1$, it follows that $\alpha=0$, i.e. $x_{r}=y_{r}$.

Hence, by induction, $\mathbf{x}=\mathbf{y}$ provided $b_{r}=1, \forall r \geqq 0$ i.e. $\Sigma_{\mathbf{a}}=\Sigma_{n}$.

(ii) Now suppose $\phi_{m / n}$ is invertible on $\Sigma_{\mathrm{a}}$. Without loss of generality, assume $\mathbf{a}=\left(m n c_{1}, m n c_{2}, \ldots\right)$ where each $c_{i}$ is an integer coprime to $m n$. Let $M$ $=|m|$ and $C_{r}=\prod_{j=1}^{r} c_{j}, C_{0}=1$.

Let $\mathbf{x}$ and $\mathbf{y}$ be in the same atom of $\vee_{-\infty}^{\infty} \phi_{m / n}^{i} P$. In particular, for each $r \geqq 0, x_{r}$ and $y_{r}$ lie in the same atom of $\omega_{C_{r}}^{-1} \bigvee_{-r}^{r} \omega_{m}^{-r-i} \omega_{n}^{-r+i} S(m)$ (compare 2.2(1)).

The atoms of $\vee_{-r}^{r} \omega_{m}^{-r-i} \omega_{n}^{-r+i} S(m) \vee S\left(n^{2 r}\right)$ are bounded by arcs of measure at most $1 / M^{2 r+1}$ (compare (2) above). Hence there are integers $t(r, i)$ for $-r \leqq i \leqq r$ with $0 \leqq t(r, i)<C_{r} M^{r+i} n^{r-i}$ such that

$$
0 \leqq\left\|x_{r}-y_{r}\right\|-t(r, i) / C_{r} M^{r+i} n^{r-i} \leqq 1 / C_{r} M^{2 r+1} .
$$

The proof proceeds in the obvious way by analogy with (i), setting $i=0, i=-r$ and $i=+r$ in (4) as required.

The results of this section are now summed up in the following statement.

THEOREM 2.4. (i) Let $\phi_{m / n}$ be an ergodic ' expansive' (i.e. $|m / n|>1$ ) endomorphism of the $n$-adic solenoid $\Sigma_{n}$. Then $\phi_{m / n}$ is a one-sided Bernoulli shift.

(ii) The ergodic automorphism $\phi_{m / n}$ of the mn-adic solenoid $\Sigma_{m n}$ is a Bernoulli shift.

Proof. (i) $P=\pi_{0}^{-1} S(m)$ is a Bernoulli generator for $\phi_{m / n}$, by 2.2 and 2.3(i).

(ii) If $|m|>n$, then $P=\pi_{0}^{-1} S(m)$ is a Bernoulli generator for $\phi_{m / n}$, by 2.2 and 2.3(ii). If $|m|<n$, then $Q=\pi_{0}^{-1} S(n)$ is a Bernoulli generator for $\phi_{n / m}$ by the same argument. But $\phi_{m / n}$ is the inverse of $\phi_{n / m}$, so they share Bernoulli generators.

3. Let $\phi_{m / n}$ now be a fixed but arbitrary ergodic automorphism of a solenoid $\Sigma_{\mathrm{a}}$, and let $\boldsymbol{M}=\max (|m|, n)$. Assume $\mathbf{a}=\left(a_{1}, a_{2}, \ldots\right)$ and set $A_{r}=\prod_{i=1}^{n} a_{i}$. For $r>0$, let $P_{r}=\phi_{A_{r}} \pi_{0}^{-1} S(M)=\pi_{r}^{-1} S(M)$. Now $\phi_{A_{r}}$ is a $\mu$-preserving endomorphism of $\Sigma_{\mathrm{a}}$, so by $2.2, P_{r}$ is a Bernoulli partition for $\phi_{m / n}$. Let $S_{r}$ be the $\sigma$-algebra generated by the partition $\vee_{-\infty}^{\infty} \phi_{m / n}^{i} P_{r}$. It follows from the proof of 2.3 (concerning $P_{0}$, and the definition of $P_{r}$, that $S_{r}$ separates points of $\Sigma_{\mathbf{a}}$ having distinct $r$ th coordinates. Hence, $\left\{S_{r}\right\}, r \geqq 0$, is a sequence of $\sigma$-algebras increasing to the full $\sigma$-algebra on $\Sigma_{\mathrm{a}}$, and $\phi_{m / n}$ restricted to each $S_{r}$ is a Bernoulli shift (with Bernoulli generator $P_{r}$ ). Hence, the automorphism $\phi_{m / n}$ of $\Sigma_{\mathrm{a}}$ is isomorphic to a generalised Bernoulli shift in the sense of D. S. Ornstein [4], and by his theorem is thus isomorphic to a Bernoulli shift with entropy $\log M$.

REMARK. Since Ornstein's theorem only applies in the invertible case, it cannot be deduced by the above method that an arbitrary expansive endomorphism of a solenoid is isomorphic to a one-sided Bernoulli shift.

\section{REFERENCES}

1. L. M. Abramov, The entropy of an automorphism of a solenoidal group, Teor. Verojatnost. i Primenen. 4 (1959), 249-254 = Theor. Probability Appl. 4 (1959), 231-236. MR 22 \#8103. 
2. S. Eilenberg and N. E. Steenrod, Foundations of algebraic topology, Princeton Univ. Press, Princeton, N. J., 1952. MR 14, 398.

3. James E. Keesling, The group of homeomorphisms of a solenoid, Trans. Amer. Math. Soc. 172 (1972), 119-131. MR 47 \#284.

4. D. S. Ornstein, Two Bernoulli shifts with infinite entropy are isomorphic, Advances in Math. 5 (1970), 339-348. MR 43 \#478a.

5. L. S. Pontrjagin, Topological groups, GITTL, Moscow, 1938; English transl., Princeton Math. Ser., vol. 2, Princeton Univ. Press, Princeton, N. J., 1939. MR 1, 44.

6. B. Weiss, The isomorphism problem in ergodic theory, Bull. Amer. Math. Soc. 78 (1972), 668-684. MR 46 \#3751.

Department of Statistics, The University, Manchester M13 9PL, England 